\title{
Formação Docente Continuada Baseada em Cursos Abertos Massivos On-line (MOOCs): Experiência da Universidade Federal de Pernambuco durante a Pandemia pelo Coronavírus
}

\author{
Josiane Lemos Machiavelli ${ }^{1}$, Patricia Smith Cavalcante ${ }^{2}$ \\ ${ }^{1}$ Pós-graduação em Educação Matemática e Tecnológica - Universidade Federal de \\ Pernambuco (UFPE) - Recife, PE - Brasil \\ josiane.machiavelli@gmail.com, patricia.cavalcante@ufpe.br
}

\begin{abstract}
This paper presents a training model based on massive open online courses, MOOCs, developed by the Federal University of Pernambuco to guide your teachers for the pedagogical use of the $G$ Suite, a set of services implemented at the university to facilitate remote activities during the period of physical distancing to reduce the coronavirus spread. Preliminary results of the research show that the suggested course model can be an efficient and effective alternative to contribute to continuing teacher education, favor cognitive and behavioral engagement, and provide multiple and flexible teaching and learning structures, capable of reaching a large number of students, with different learning styles and training needs.
\end{abstract}

Resumo. Este artigo apresenta um modelo de formação baseado em Cursos Abertos Massivos On-line (MOOCs), proposto pela Universidade Federal de Pernambuco para orientar seus docentes no uso pedagógico do G Suite, conjunto de serviços implantado na instituição para ser utilizado nas atividades remotas durante o período de distanciamento físico para enfrentamento do coronavírus. Resultados preliminares da pesquisa apontam que o modelo de curso sugerido pode ser uma alternativa eficiente e eficaz para contribuir com a formação docente continuada, favorece o engajamento cognitivo e comportamental, e proporciona estruturas múltiplas e flexíveis de ensino e aprendizagem, capazes de alcançar grande número de professores, com diferentes estilos de aprendizagem e necessidades formativas.

\section{Introdução}

Este artigo apresenta a experiência da Universidade Federal de Pernambuco (UFPE) no planejamento, desenvolvimento e na oferta de um curso baseado em cursos massivos on-line - Massive Open Online Courses (MOOCs) para oportunizar a formação dos seus docentes no uso pedagógico do G Suite, conjunto de serviços ofertados pela Google implantado na instituição e que está sendo utilizado nas atividades remotas durante o período de distanciamento físico, necessário para enfrentamento do coronavírus.

A formação docente no Brasil é alvo de críticas há anos. Estudos mostram que é muito comum os professores chegarem às instituições de ensino despreparados para 
atuar [Barreto 2015], principalmente quando se fala em ensino superior. Apesar de boa parte dos professores terem realizado sua formação em cursos de pós-graduação stricto sensu e possuírem experiência profissional relevante, ainda é comum o despreparo para lidar com os processos de ensino e aprendizagem [Almeida 2012]. Se levarmos em consideração as mudanças ocorridas na sociedade com o incremento do uso das Tecnologias Digitais da Informação e Comunicação (TDICs), os problemas ficam ainda mais sérios, pois elas ainda são pouco exploradas nas formações, fazendo com que muitos professores sejam resistentes ou não se sintam confortáveis para utilizá-las como ferramentas pedagógicas.

Tais fatos foram evidenciados no momento de pandemia pelo coronavírus. Em questão de dias, as TDICs passaram a ser uma das poucas alternativas viáveis para que as instituições de ensino continuassem ou retomassem suas atividades. Com isso, vieram à tona muitos dos problemas já conhecidos e que agora, obrigatoriamente, devem ser enfrentados, como a falta de intimidade de muitos docentes para utilizar os recursos tecnológicos digitais na prática profissional. Mais do que inserir as tecnologias no dia a dia, diversos gestores e professores estão precisando aprender ou aprofundar seus conhecimentos sobre o bom uso pedagógico desses recursos. Nesse sentido, estratégias têm sido implementadas a fim de dar suporte a esses docentes de modo que se sintam mais confortáveis a seguir com suas atividades de modo remoto.

Os MOOCs, originalmente, são cursos on-line, projetados para atender um grande número de participantes, que podem ser acessados por qualquer pessoa, em qualquer lugar, desde que haja conexão com a Internet, sem restrições de qualificação prévia. Tais cursos surgiram com a proposta de oferecer uma experiência de ensino e aprendizagem completa, on-line e gratuitamente. Entretanto, já existem iniciativas de uso com números reduzidos de estudantes, cursos não totalmente abertos nem gratuitos, aplicados à educação corporativa e como apoio ao ensino presencial [Atiaja e Proenza 2016; Yousef et al. 2014]. Compreendemos que este modelo de curso pode ser uma alternativa viável para condução dos processos formativos docentes, especialmente nesse momento de pandemia.

No entanto, apesar da ampla utilização em diversos países e diferentes contextos, a literatura apresenta uma série de problemas e desafios em relação aos MOOCs, como: altas taxas de evasão; falta de qualidade no Design Educacional (DE); não utilização de conceitos essenciais do DE na sua produção; o monitoramento das ofertas educacionais não costuma ser efetivo; em muitas ofertas as avaliações de aprendizagem não passam de simples testes, pouco desafiadoras e descontextualizadas das necessidades práticas dos cursistas; inúmeros cursos não estimulam o contato e as trocas entre os participantes; não há padrões suficientes que possibilitem avaliar a qualidade pedagógica dos cursos; é comum não haver professores ou facilitadores que estimulem a participação dos estudantes ou estejam disponíveis para esclarecer dúvidas; a comunicação síncrona é pouco utilizada; ainda faltam pesquisas que investiguem melhor quem são os participantes, o que motiva as pessoas a participarem de MOOCs e concluírem seus estudos nesse formato de curso [Atiaja e Proenza 2016; Dillenbourg et al. 2014; Mcguire, Raaper e Nikolova 2016; Siemens 2013]. O modelo de curso apresentado neste artigo busca minimizar esses problemas e contribuir com uma formação docente ancorada em princípios que envolvem a aprendizagem flexível, prática, aplicada e colaborativa. 


\section{A UFPE no contexto da pandemia pelo coronavírus}

A UFPE suspendeu suas atividades acadêmicas presenciais em março de 2020 e não há previsão, até o momento, de retomada, a exemplo do que acontece com muitas instituições de ensino em todo o mundo (UNESCO, c2020). No entanto, a Universidade tem se empenhado na busca de alternativas que garantam a continuidade de pelo menos parte das atividades de modo on-line. Muitos cursos de pós-graduação, por exemplo, voltaram a funcionar remotamente a partir de junho de 2020 e a retomada de parte das atividades de graduação está prevista para acontecer em agosto de 2020. Para que isso seja possível, além de disponibilizar soluções tecnológicas que viabilizem a realização de atividades on-line, como é o caso do G Suite, viu-se a necessidade de oferecer curso de formação que oriente o bom uso desses recursos pelos servidores, começando pelos mais de dois mil e quinhentos docentes [UFPE 2019].

\section{Formação G Suite para Servidores da Universidade Federal de Pernambuco}

O curso baseia-se na aprendizagem flexível on-line, aqui entendida como um processo de ensino e aprendizagem centrado nas necessidades dos cursistas, que oferece variadas opções de estudo para atender diferentes perfis de estudantes, como flexibilidade de horário e de locais (mesmo que virtuais) onde os estudos são desenvolvidos, recursos e abordagens pedagógicas utilizadas, que podem ser síncronas e assíncronas. Do ponto de vista de interação, as pedagogias flexíveis proporcionam momentos para realização de atividades individuais e coletivas, em formatos variados, como reuniões, apresentações de trabalhos, produção de conteúdos, resolução de exercícios, debates, entre outras [Huang et al. 2020a; Huang et al. 2020b]. Acredita-se que esses aspectos motivam a proatividade e independência dos cursistas e podem contribuir com um aprendizado mais fácil, engajado e eficaz.

O desenho da formação foi feito a partir de componentes que se enquadram em três categorias [Filatro 2008; Huang et al. 2020a]: i) pré-análise envolveu conhecer o perfil dos cursistas, identificar os recursos tecnológicos disponíveis e a definição dos objetivos de aprendizagem a serem desenvolvidos no curso; ii) design, desenvolvimento e implementação, que diz respeito à definição das estratégias pedagógicas, identificação de REAs, produção ou adaptação de materiais didáticos e disponibilização do curso no Google Classroom; iii) avaliação, que engloba tanto as atividades desenvolvidas pelos estudantes ao longo do curso quanto a avaliação final do curso pelos discentes e equipe envolvida a fim de identificar pontos de melhoria.

Para auxiliar no planejamento da formação, o primeiro passo foi conhecer a experiência dos professores da UFPE no uso de recursos tecnológicos digitais na sua prática profissional. Para isso, foi feita pesquisa, com apoio do recurso "Formulários Google", da qual participaram 675 professores. Os resultados revelaram que 97\% dos docentes utilizam algum tipo de recurso tecnológico digital no dia a dia profissional. No entanto, apenas $29 \%$ utilizam ambientes virtuais de aprendizagem, como o Moodle ou Google Classroom. Os recursos mais utilizados costumam ser as ferramentas de comunicação (como mensagens instantâneas e e-mail) e os editores de textos, apresentações e planilhas. Outros dados importantes: 54\% dos professores informaram que não se sentem preparados para utilização desses recursos na prática profissional; $77 \%$ dos respondentes informaram que não sabem o que são Recursos Educacionais 
Abertos (REAs), e apenas 8\% declararam que utilizam REAs nas atividades docentes. Quando questionados sobre a participação prévia em processos formativos que orientam o uso das tecnologias digitais para apoio aos processos de ensino e aprendizagem, apenas $27 \%$ dos professores informaram que já participaram de algum tipo de formação.

A partir desses dados, viu-se que a formação que estava sendo proposta deveria contemplar duas perspectivas: i) uma instrumental, que envolve a apresentação das soluções tecnológicas disponíveis no G Suite; ii) outra pedagógica, que engloba a discussão das formas de incluí-las nos processos de ensino e aprendizagem. A seguir, apresentamos os princípios que nortearam o desenvolvimento do "Curso de Formação G Suite para Servidores da Universidade Federal de Pernambuco", bem como a estrutura da formação, que vem sendo conduzida pela Secretaria de Programas em Educação Aberta e Digital da Universidade Federal de Pernambuco - Spread/UFPE.

\subsection{Princípios de design que nortearam o desenvolvimento do curso}

Os princípios de design considerados no desenvolvimento do curso podem ser divididos em três grandes categorias:

1) Currículo e espaço virtual de aprendizagem: Tem relação com a definição dos recursos tecnológicos utilizados e o desenho do curso no ambiente virtual de aprendizagem. Decidiu-se pela estruturação do curso no Google Classroom. Embora não seja uma plataforma para oferta de cursos massivos, é o recurso que os cursistas terão à disposição na UFPE para sua prática docente, logo, entendemos que o fato de a formação acontecer nesse espaço seria uma oportunidade de aprender a utilizar o recurso por meio da exploração do próprio curso.

A flexibilidade está presente na liberdade que os cursistas têm para definir seu itinerário de estudos, respeitando seus interesses, necessidades e ritmo. Ou seja, o curso não é linear ou sequencial. Os estudantes podem seguir o roteiro proposto ou então definir seu próprio roteiro, acessando os materiais conforme suas necessidades de aprendizagem, pois uma unidade didática não é pré-requisito para acesso às outras. Os conteúdos são divididos em seis unidade didáticas, que totalizam 20 horas-aula. São elas (UFPE, 2020): i) Sobre a docência on-line (2h); ii) Como criar meu Google Drive (3h); iii) Como produzir e compartilhar meus documentos (1h); iv) Como criar uma apresentação em slides (3h); v) Como criar um formulário (3h); vi) Como criar minha Sala de Aula Google (8h). Em cada unidade são apresentados os objetivos de aprendizagem que se pretende atingir, o tempo recomendado para estudo, as atividades propostas e dicas que buscam otimizar os estudos.

Os cursistas são estimulados a expor suas dúvidas na área de comentários disponível na sala de aula virtual, que funciona de modo parecido com os fóruns de discussão. Isso favorece a interação e a troca de conhecimentos entre os próprios cursistas e destes com os docentes que mediam a formação. É disponibilizado certificado de conclusão de curso àqueles que participarem da atividade final, que envolve a estruturação de uma sala de aula no Google Classroom. Para o desenvolvimento e a oferta do curso, conta-se com apoio de equipe multidisciplinar, que envolve profissionais com experiência em Educação, Informática, Design Educacional e Supervisão Acadêmica.

2) Objetos de aprendizagem e atividades: Essa categoria envolve as características e os cuidados necessários na definição dos materiais didáticos e 
atividades da formação. Optou-se pela utilização de REAs, o que reduziu de maneira significativa o tempo dispendido para o desenvolvimento do curso e, ao mesmo tempo, favoreceu a disponibilização de conteúdos de qualidade e que atenderam ao planejamento inicial do curso, além de uma diversidade de materiais didáticos. Novos materiais precisaram ser produzidos, mas usando como referências REAs previamente identificados. A variedade de formatos de materiais didáticos busca atender diferentes estilos de aprendizagem. São disponibilizados vídeos, tutoriais, infográficos e cartões com síntese de conteúdos. A maioria dos vídeos são de curta duração (menos que 10 minutos). Muitos dos materiais estão disponíveis para download, de modo que os estudantes poderão ter acesso mesmo após o término da formação.

Cada unidade didática dispõe de uma atividade, de natureza formativa, baseada em situações reais, que estimula os cursistas a organizarem sua rotina profissional com apoio do G Suite, ou seja, possibilitam a aplicação prática dos conhecimentos adquiridos durante os estudos. O foco, nas atividades, não é definir se estão sendo feitas de modo correto ou incorreto, tanto é que não valem nota e a maioria delas não são obrigatórias. A intenção é provocar os cursistas a repensarem sua prática docente, de modo que as atividades sejam úteis no seu dia a dia. Foram programadas pelo menos duas reuniões on-line com os cursistas, os meets. Previamente agendados, não são aulas expositivas. São momentos planejados para debater as diferenças entre a docência presencial e on-line, as estratégias pedagógicas possíveis com o G Suite, e outras dúvidas que surgem durante a formação. Ao final do curso, é disponibilizada uma pesquisa de opinião a fim de conhecer as impressões dos cursistas e professores, que serão úteis para adequação da formação para oferta de novas turmas, bem como para o desenvolvimento de outros cursos.

3) Suporte e comunicação: Equipe de supervisão acadêmica e professores fica disponível durante a formação para responder às demandas dos cursistas, sejam elas de ordem técnica ou relacionadas com os conteúdos trabalhados, tendo prazo de até 24 horas para responder aos questionamentos recebidos. Foi desenvolvido um Frequently Asked Questions (FAQ), a partir das dúvidas mais recorrentes dos discentes. Isso otimizou o tempo de resposta da equipe, bem como reduziu a quantidade de demandas, pois os cursistas foram orientados a consultar o FAQ antes de acionar a equipe de professores e supervisão acadêmica.

\section{Resultados preliminares}

Enquanto este artigo foi escrito, estávamos concluindo a oferta da primeira das oito turmas previstas. Com 134 cursistas, a oferta envolveu docentes vinculados a programas de pós-graduação da UFPE, de diferentes áreas do conhecimento. Embora boa parte das atividades da formação fossem opcionais, apenas $17 \%$ dos cursistas não participaram de, pelo menos, uma parte delas, e 70\% deles finalizaram o curso de modo satisfatório, estando aptos a receber o certificado de conclusão de curso, um número muito expressivo se considerarmos que a literatura registra taxa de evasão superior a $80 \% \mathrm{em}$ MOOCs [Atiaja e Proenza 2016; Dillenbourg et al. 2014]. Outro dado que chamou atenção foi a participação assídua dos docentes nos meets temáticos e a demanda por mais encontros além dos dois inicialmente previstos.

Ao final da formação, os cursistas foram convidados a preencher um questionário de avaliação, disponibilizado no "Formulários Google", respondido por 
100 pessoas. Destas, 99\% consideraram que os conteúdos apresentados na formação importantes ou muito importantes para a prática profissional; $99 \%$ concordam, total ou parcialmente, que aprenderam a interagir rapidamente com os recursos didáticos do curso; $93 \%$ concordam, total ou parcialmente, que a forma como o curso foi estruturado facilita a aprendizagem e $99 \%$ desejam participar de outras formações que abordem o uso de recursos tecnológicos digitais na prática profissional. Chamam atenção, também, outros feedbacks apresentados pelos cursistas, como: i) o reconhecimento da importância do curso para incrementar a prática docente; ii) a indicação de que o curso contribuiu para que alguns professores se sentissem mais seguros para utilizar tecnologias digitais no dia a dia profissional; iii) o interesse demonstrado pela continuidade dos processos formativos dessa natureza. Alguns deles chegaram a sugerir, por exemplo, uma política de educação permanente para professores da UFPE.

Das críticas observadas em relação ao curso, a análise dos comentários deixados pelos cursistas na pesquisa de opinião e também nas conversas durante a oferta da turma, possibilita concluir que a maioria delas diz respeito à pouca familiaridade com o G Suite, falta de estudo dos materiais disponibilizados na formação ou tentativa de querer enquadrar a formação nas características do ensino tradicional presencial. Não são, portanto, problemas relacionados aos recursos tecnológicos utilizados, desenho da sala de aula ou atuação da equipe de suporte acadêmico. Uma crítica que merece ser analisada com cuidado pela equipe responsável pela produção do curso diz respeito à carga horária e duração. Alguns participantes consideram que 20 horas não corresponde ao tempo que eles dispenderam para realizar a formação e que duas semanas foram insuficientes para adequado desenvolvimento dos estudos e atividades sugeridas. Estimar a carga horária em cursos on-line não é uma tarefa fácil, pois é influenciada por diversos fatores, como a complexidade dos conteúdos apresentados no curso, o perfil dos cursistas e a quantidade de objetos de aprendizagem disponibilizados. Considerando a pouca familiaridade dos cursistas com ambientes virtuais de aprendizagem e recursos do G Suite, é provável que isso tenha influenciado a percepção de que precisaram de um tempo superior às 20 horas inicialmente previstas para concluir os estudos.

\section{Considerações finais}

Os dados até aqui analisados demonstram que os cursos baseados em MOOCs podem ser alternativas eficientes (compreendidas como sendo capazes de atingir os objetivos propostos com rapidez, qualidade, baixo custo e atendendo a um grande número de pessoas) e eficazes (apresentam resultados satisfatórios) para a formação docente continuada. Quando adequadamente planejados para atender às reais necessidades dos cursistas, facilitam o engajamento cognitivo, reduzindo o esforço e o tempo necessários para aprender e realizar determinadas atividades, e comportamental, visto pela excelente adesão às atividades propostas, bem como ampliam as oportunidades de aprendizagem. Ainda, esse modelo de curso oferece estruturas múltiplas e flexíveis de ensino e aprendizagem, que, além de alcançar milhares de pessoas, conseguem atender a diferentes interesses e estilos de aprendizagem, algo difícil de acontecer apenas por meio do ensino presencial. O curso, mesmo sendo de curta duração, também conseguiu fomentar a reflexão sobre as inúmeras possibilidades de desenhos de aulas quando estas são mediadas por tecnologias digitais. 


\section{Referências}

Almeida, M. I. de. (2012) "Formação do professor do ensino superior: desafios e políticas institucionais”. São Paulo: Cortez.

Atiaja, L. N. A., Proenza, R. S. G. (2016) "MOOCS: origin, characterization, principal problems and challenges in higher education". Journal of e-Learning and Knowledge Society, v. 12, n. 1, p. 65-76.

Barretto, E. S. de S. (2015) "Políticas de formação docente para a educação básica no Brasil: embates contemporaneous". Revista Brasileira de Educação, v. 20, n. 62, p. 679-701.

Dillenbourg, P. et al. (2014) "Massive Open Online Courses: current state and perspectives". Proceedings of the Dagstuhl Perspectives Workshop 2014. Wadern: [s.n.].

Filatro, A. (2008) "Design instrucional na prática”. São Paulo: Pearson Education do Brasil.

Huang, R.H. et al. (2020a) "Guidance on flexible learning during campus closures: ensuring course quality of higher education in COVID-19 outbreak". Beijing: Smart Learning Institute of Beijing Normal University.

Huang, R.H. et al. (2020b) "Handbook on facilitating flexible learning during educational disruption: the chinese experience in maintaining undisrupted learning in COVID-19 outbreak". Beijing: Smart Learning Institute of Beijing Normal University.

Mcguire, W., Raaper, R., Nikolova, V. (2016) "Three perspectives on hybridizing x and cMOOCs to create an online course on digital CVs". International Multidisciplinary Journal, v. 3, n. 2, p. 20-33.

Siemens, G. (2013) "Massive open online courses: innovation in education?" In: Mcgreal, R.; Kinuthia, W.; Marshall, S. (Org.). Open Educational Resources: innovation, research and practice. Athabasca: Commonwealth of Learning, Athabasca University, p. 5-15.

United Nations Educational, Scientific and Cultural Organization - UNESCO (c2020) "Educação: da interrupção à recuperação". Disponível em: https://pt.unesco.org/covid19/educationresponse. Acesso em: 31 jul 2020.

Universidade Federal de Pernambuco - UFPE (2019) "UFPE em números". Disponível em: https://www.ufpe.br/institucional/ufpe-em-numeros. Acesso em: 28 mai 2020.

Universidade Federal de Pernambuco - UFPE (2020) “Apresentação do Curso de Formação G Suite para Servidores da Universidade Federal de Pernambuco. UFPE: Secretaria de Programas em Educação Aberta e Digital - Spread/UFPE.

Yousef, A. M. F. et al. (2014) "MOOCs: a review of the state-of-the-art". Proceedings of the 6th International Conference On Computer Supported Education 2014, Barcelona: [s.n.], p. 9-20. 